\title{
Resistance of Klebsiélla Species Isolates From Two Institutions in Nairobi, Kenya, to Commonly Prescribed Antimicrobial Agents
}

\author{
J.M. BURURIA* ${ }^{1}$, P.N. KINYANJUI ${ }^{2}$, P.G. WAIYAKI ${ }^{3}$ AND S.M. KARIUKI $^{3}$ \\ 'Department of Pharmaceutics and Pharmacy Practice, University of Nairobi, P.O. Box 19676-00202, \\ Nairobi, Kenya \\ ${ }^{2}$ Department of Biochemistry, University of Nairobi, P.O. Box 30197-00100, Nairobi, Kenya \\ ${ }^{3}$ Centre for Microbiology Research, Kenya Medical Research Institute, P.O. Box 19463-00202, Nairobi, \\ Kenya
}

\begin{abstract}
Klebsiella species isolates collected from the Kenyatta National Hospital and the Centre for Microbiology Research of the Kenya Medical Research Institute in Nairobi, Kenya were identified and screened for resistance to commonly prescribed antimicrobial agents. Most of the isolates were found to exhibit resistance to more than three agents amongst those tested by both disk diffusion tests and minimum inhibitory concentration determination.
\end{abstract}

Key words: Klebsiella, resistance, minimum inhibitory concentration, disk diffusion

\section{INTRODUCTION}

The Klebsiella species have been shown to be important opportunistic pathogens featuring prominently among causative agents of nosocomial infections [1] and community acquired bacteraemia as well as infections at other sites [2]. Epidemics caused by antimicrobial resistant Klebsiella species have led to closures of hospital specialist units or even whole hospitals [3]. At the Kenyatta National Hospital, the major public referral and teaching hospital in Kenya, high prevalence of infection with Klebsiella species has been reported [4-5]. The current study was undertaken to find out the prevalence of resistance to antibimicrobial agents used in the treatment of urinary and non-urinary isolates collected at the Kenyatta National Hospital and at the Centre for Microbiology Research (CMR) of the Kenya Medical Research Institute.

\section{EXPERIMENTAL}

\section{Samples}

Samples of urine, blood, cerebrospinal fluid, sputum, stool and pus as well as vaginal and throat swabs were examined for the presence of Klebsiella species at the routine Microbiology
Laboratory of the Department of Medical Microbiology of the University of Nairobi (based at the Kenyatta National Hospital) and at the CMR of the Kenya Medical Research Institute between October 1991 and March 1992. A total of 118 isolates were obtained from samples from the Kenyatta National Hospital and the CMR. An additional 16 isolates that had already been identified were obtained from the Kenya Medical Research Institute/Wellcome Trust Research Laboratory (KEMRI/WTRL), Nairobi. The isolates consisted of 86 urinary and 48 non-urinary isolates. These were subjected to disk diffusion tests at the Kenyatta National Hospital and the CMR laboratories and minimum inhibitory concentration (MIC) determination at the KEMRI/WTRL.

\section{Disk diffusion tests}

The tests were carried out with a bacterial cell suspension adjusted to a turbidity comparable to that of a 0.5 McFarland turbidity standard (approximately $10^{8}$ colony forming units per ml).Urinary isolates were tested with disks containing predetermined amounts of ampicillin $(10 \mu \mathrm{g})$, co-trimoxazole $(1.25 / 23.75 \mu \mathrm{g})$, gentamicin $(10 \mu \mathrm{g})$, nalidixic acid $(30 \mu \mathrm{g})$, nitrofurantoin $(300 \mu \mathrm{g})$, sulphamethoxazole (300 $\mu \mathrm{g})$, streptomycin $(10 \mu \mathrm{g})$ and tetracycline (30

*Author to whom correspondence may be addressed. 
$\mu \mathrm{g})$. Non-urinary isolates were tested with disks containing ampicillin $(10 \mu \mathrm{g})$, chloramphenicol (30 $\mu \mathrm{g})$, co-trimoxazole $(1.25 / 23.75 \mu \mathrm{g})$, gentamicin $(10 \mu \mathrm{g})$, kanamycin $(30 \mu \mathrm{g})$, streptomycin $(10 \mu \mathrm{g})$, sulphamethoxazole (300 $\mu \mathrm{g})$ and tetracycline $(30 \mu \mathrm{g})$. Any isolate that appeared resistant to four or more antimicrobial agents was tested with single disks containing amikacin (30 $\mu \mathrm{g})$, aztreonam $(30 \mu \mathrm{g})$, cefotaxime $(30 \mu \mathrm{g})$, ceftazidime $(30 \mu \mathrm{g})$, ceftriaxone $(30 \mu \mathrm{g})$, cefuroxime $(30 \mu \mathrm{g})$, or enoxacin $(10 \mu \mathrm{g})$.

\section{Determination of Minimum Inhibitory Concentrations}

Eighty six isolates picked at random from among the 134 originally collected were used for the determination of the MIC by the agar dilution method on Drug Sensitivity Testing (DST) agar (Oxoid Ltd, Basingstoke, Hampshire, England). The MICs of amoxycillin, co-amoxiclav, chloramphenicol, ciprofloxacin, gentamicin, streptomycin, sulphamethoxazole, tetracycline, trimethoprim, and co-trimoxazole against the 86 isolates were determined. Escherichia coli strain ATCC 25922 obtained from the National Public Health Laboratories, Nairobi, Kenya was used as the reference strain for all MIC determinations.

\section{RESULTS}

\section{Identification and distribution of Klebsiella isolates from various specimens and swabs}

The 134 isolates were identified as $K$. pneumoniae subspecies pneumoniae, and ozaenae or $K$ oxytoca. One hundred and seventeen isolates $(87.3 \%)$ were $K$ pneumoniae subspecies pneumoniae, while fifteen isolates $(11.2 \%)$ were $K$. oxytoca and two isolates (1.5 $\%$ ) were $K$ pneumoniae subspecies ozaenae.

\section{Disk diffusion tests}

Table 1 shows the resistance levels of the Klebsiella isolates to each of the drugs used in the disk diffusion test. It was found that all 86 urinary isolates $(100 \%)$ were resistant to ampicillin. Resistance to the other drugs among urinary isolates was found to rise in the order of nalidixic acid (3.5\%), nitrofurantoin $(9.3 \%)$, gentamicin $(66.0 \%)$, tetracycline $(77.9 \%)$, streptomycin $(90.7 \%)$, co-trimoxazole $(96.5 \%)$, and sulphamethoxazole $(97.7 \%)$.

Among the non-urinary isolates, resistance was observed in the increasing order of kanamycin $(47.9 \%)$, gentamicin $(52.1 \%)$, chloramphenicol $(66.7 \%)$, streptomycin and tetracycline $(68.8$ $\%$ ), co-trimoxazole $(72.9 \%)$, sulphamethoxazole $(85.4 \%)$ and ampicillin $(97.9 \%)$. The isolate that was susceptible to ampicillin was one of the two Klebsiella pneumoniae subspecies ozaenae isolates encountered in this study. Besides the results presented in Table 1 . two isolates were found to be resistant to ceftriaxone, but not to amikacin, aztreonam, cefotaxime, ceftazidime, cefuroxime or enoxacin.

Table 2 shows a comparison of the susceptibilities of urinary and non-urinary isolates to the various antimicrobial agents used. Similar antimicrobial agents were used for both groups of isolates except that nitrofurantoin and nalidixic acid were included only for urinary isolates while chloramphenicol and kanamycin were tested on non-urinary isolates only.

For both the urinary and non-urinary categories, it was observed that most of the isolates were resistant to ampicillin, sulphamethoxazole and co-trimoxazole. Additionally, there was no significant difference in resistance prevalence between the urinary and non-urinary isolates. 
Table 1: Number of isolates resistant to each antimicrobial agent as determined by disk diffusion sensitivity tests.

\begin{tabular}{lcc}
\hline Antimicrobial agent & $\begin{array}{l}\text { Number resistant } \\
\text { urinary isolates (\%) }\end{array}$ & $\begin{array}{l}\text { Number resistant } \\
\text { non-urinary isolates (\%) }\end{array}$ \\
\hline Ampicillin & $\mathbf{8 6 ( 1 0 0 )}$ & $47(97.9)$ \\
Sulphamethoxazole & $\mathbf{8 4}(97.7)$ & $41(85.4)$ \\
Co-trimoxazole & $83(96.5)$ & $35(72.9)$ \\
Streptomycin & $78(90.7)$ & $35(72.9)$ \\
Tetracycline & $67(77.9)$ & $33(68.8)$ \\
Chloramphenicol & - & $32(66.7)$ \\
Gentamicin & $57(66.3)$ & $25(52.1)$ \\
Kanamycin & - & $23(47.9)$ \\
Nitrofurantoin & $\mathbf{8}(9.3)$ & - \\
Nalidixic acid & $3(3.5)$ & - \\
\hline \multicolumn{2}{c}{ Not tested in these isolates } &
\end{tabular}

Table 2: Comparison of prevalence of resistance to various antimicrobial agents tested against urinary and non-urinary Klebsiella isolates

\begin{tabular}{|c|c|c|c|c|}
\hline \multirow[t]{2}{*}{ Antimicrobial agent } & \multicolumn{2}{|c|}{ Percentage isolates } & \multirow{2}{*}{$\begin{array}{c}\chi^{2} \\
\text { Yate's } \\
\text { Corrected }\end{array}$} & \multirow[t]{2}{*}{ p-value } \\
\hline & Urinary & Non-urinary & & \\
\hline Ampicillin & 100 & 97.9 & 0.01 & 0.9199 \\
\hline Gentamicin & 66.3 & 52.1 & 2.86 & 0.0906 \\
\hline Streptomycin & 90.7 & 72.9 & 3.52 & 0.0605 \\
\hline Sulphamethoxazole & 97.7 & 85.4 & 1.57 & 0.2097 \\
\hline Co-trimoxazole & 96.5 & 72.9 & 6.22 & 0.0126 \\
\hline Tetracycline & 77.9 & 68.8 & 0.87 & 0.3507 \\
\hline
\end{tabular}

$p \leq 0.01$ is significant.

Table 3: The $\mathrm{MIC}_{50}, \mathrm{MIC}_{90}$ and $\mathrm{MIC}$ range of antimicrobial agents against 86 Klebsiella isolates and the resistance prevalence to each agent.

\begin{tabular}{lcccc}
\hline Antimicrobial Agent & $\begin{array}{l}\mathbf{M I C}_{\mathbf{5}} \\
(\mu \mathrm{g} / \mathbf{m})\end{array}$ & $\begin{array}{l}\mathbf{M I C}_{\mathbf{9 0}} \\
(\mu \mathrm{g} / \mathbf{m})\end{array}$ & $\begin{array}{l}\text { MIC range } \\
(\mu \mathrm{g} / \mathbf{m l})\end{array}$ & $\begin{array}{l}\text { Number of } \\
\text { resistant isolates (\%) }\end{array}$ \\
\hline Amoxycillin & 128 & 128 & $1-128$ & $85(98.8)$ \\
Sulphamethoxazole & $>1024$ & $>1024$ & $16->1024$ & $76(88.4)$ \\
Co-trimoxazole & $>32 / 640$ & $>32 / 640$ & $0.25 / 5->32 / 640$ & $67(77.9)$ \\
Trimethoprim & 128 & $>256$ & $0.25->256$ & $67(77.9)$ \\
Tetracycline & 64 & 128 & $2-128$ & $65(75.6)$ \\
Chloramphenicol & 64 & 128 & $1-128$ & $62(72.1)$ \\
Gentamicin & 32 & 128 & $1-128$ & $53(61.6)$ \\
Co-amoxiclav & $32 / 16$ & $32 / 16$ & $1 / 0.5-64 / 32$ & $46(53.5)$ \\
Ciprofloxacin & 0.06 & 0.25 & $0.03-1$ & $0(0.0)$ \\
\hline
\end{tabular}




\section{Minimum inhibitory concentrations.}

The minimum inhibitory concentration (MIC) of 9 antimicrobial agents was determined against 86 Klebsiella isolates picked at random from among both urinary and non-urinary isolates. The $\mathrm{MIC}_{50}$ and $\mathrm{MIC}_{90}$ were considered an indication of the overall response of the 86 isolates to each antimicrobial agent (Table 3). The values of both $\mathrm{MIC}_{50}$ and $\mathrm{MIC}_{90}$ for all the agents tested except ciprofloxacin were above the MIC break points for resistance as defined by the National Committee for Clinical Laboratory Standards [6].

From the results shown in Table 3 it is clear that the smallest number of resistant isolates was observed with co-amoxiclav $(53.5 \%)$ while the highest was observed with amoxycillin $(98.8 \%$ ). It was observed that resistance to trimethoprim and to co-trimoxazole appeared in a similar number of isolates $(77.9 \%)$ while resistance to sulphamethoxazole alone appeared in a higher number of isolates $(88.4 \%)$. Further, all the isolates susceptible to trimethoprim were also found to be susceptible to co-trimoxazole.

\section{DISCUSSION}

The prevalence of strains in this study reflects a trend in which Klebsiella pneumoniae subspecies pneumoniae is reported to be responsible for more infections than Klebsiella oxytoca and the other subspecies put together [7].

Disk diffusion sensitivity tests showed that prevalence of resistance was above $66 \%$ for all the drugs tested except for nalidixic acid and nitrofurantoin among the urinary isolates and for gentamicin and kanamycin among the nonurinary isolates. All except 8 among the nonurinary isolates were resistant to at least three drugs. The same trend was observed when the minimum inhibitory concentrations were determined.

All drugs were tested in concentrations up to at least three MIC levels above the National Committee for Clinical Laboratory Standards defined break points for resistance [6]. The
$\mathrm{MIC}_{50}$ and $\mathrm{MIC}_{90}$ of all the drugs tested except that of ciprofloxacin were at least two levels above the NCCLS break points. These results show that most of the Klebsiella isolates dealt with in this study exhibited very high levels of resistance to the antimicrobial agents tested. Moreover, they exhibited multiple resistance to the agents. The isolates were, however, susceptible to amikacin, aztreonam, enoxacin, and the third generation cephalosporins. Only two isolates were confirmed to have been resistant to ceftriaxone. The presence of those two isolates resistant to ceftriaxone raises the possibility of the presence of extended spectrum beta-lactamases among the isolates. An extended-spectrum beta-lactamase producing Klebsiella pneumoniae among neonatal pathogens has been reported previously [8]. It has also been reported that resistance to third generation cephalosporins may exist and yet not be shown by routine disk sensitivity tests unless disks containing clavulanic acid are included to reveal such resistance during routine testing [9]. It would thus be important to include these disks in all tests involving cephalosporins as a surveillance strategy against development of extended spectrum of beta-lactamases.

The very high levels of resistance to gentamicin, chlorampenicol, co-timoxazole, trimethoprim and sulphamethoxazole show that these agents have a limited capacity to treat infections caused by Klebsiella species. It has been reported that nitrofurantoin does not share cross-resistance with commonly prescribed antimicrobial agents. It can be used for the empirical eradication of uropathogens [25].

All isolates were susceptible to ciprofloxacin, a fluoroquinolone. Unlike the first generation quinolones represented by nalidixic acid, fluoroquinolone are reported to have a lower propensity for the development of resistance and to have superior activity against uropathogens in comparison to co-trimoxazole [10-11]. Moreover, because of the continued development of compounds with improved antimicrobial spectra and pharmacokinetics, the fluoroquinolones could become the major antibacterial agents of the $21^{\text {st }}$ century. However, they should be used in an educated 
fashion, based on a careful balance between their ease of use versus the risk of emerging resistance and toxicity [11-12].

\section{REFERENCES}

[1] W. H. Traub, I. Schwarze and D. Bauer, Chemother., 46 (2000) 1-14.

[2] J. E. McGowan Jr, Rev. Infect. Dis., 7 (1985) 357-370.

[3] M. W.Casewell and I. Phillips, Am. J. Med. 70 (1981) 459-462.

[4] J. N. Muthotho, P. G.Waiyaki, M. Moninda and J. Karuri, Programme Abstracts, International Congress for Infectious Diseases, Montreal, Canada, 1990. Abstract No. 569.

[5] N.B. Mirza, I. A.Wamola and J. Bubi, Medicom 3 (1981) 105-107.

[6] National Committee for Clinical Laboratory Standards, Methods for Dilution Antimicrobial Susceptibility
Tests for Bacteria That Grow Aerobically, $5^{\text {th }}$ Ed., Villanova, Pa, USA 1998.

[7] S. Chamberland, J. Ecuyer, C. Lessard, M. Bernier, P. Provencher, M. G. Bergeron and The Canadian Study Group, Clin. Infect. Dis. 15 (1992) 615-628.

[8] S. Kariuki, J. E. Corkill, G. Revathi, R. Musoke and C.A. Hart, Antimicrob. Agents Chemother., 45 (2001) 21412143.

[9] E. Stürenburg, M. Lang, M. A. Horstkotte, R. Laufs and D. Mack, J. Antimicrob. Chemother., 54 (2004) 870875.

[10] T. M. Hooton, Infect. Dis. Clin. North Am. 17 (2003) 303-332.

[11] A. M. Emmerson and A. M. Jones, J. Antimicrob. Chemother. 51 Suppl 1 (2003)13-20.

[12] F. Van Bambeke, J. M. Michoti, J. Van Eldere and P. M. Tulkens, Clin. Microbiol. Infect., 11(2005) 256- 280. 